\title{
HIGHLIGHTS
}

EPILEPSY

\section{Nonsynaptic mechanisms for interictal discharges in drug-intractable epilepsy}

Roopun et al. have identified a nonsynaptic mechanism that is responsible for interictal discharges in drug-intractable epilepsy. Their findings might explain why drugs are ineffective in such cases, and could aid the development of new treatments.

\section{$44 . . . V F 0$ s occurred at the onset of interictal events ... this leads to "a state of pathological hyperexcitability" 77}

Epilepsy treatment typically involves pharmacological intervention to target chemical signals associated with interictal activity. In around $30 \%$ of patients, however, drug treatments are ineffective, leaving surgery as the only option. By studying the mechanisms that cause epileptiform activity in the cortex of these patients, insights might be gained into why drugs often fail to prevent seizures.

Very fast oscillations (VFOs) are highfrequency brain waves associated with interictal activity. In vitro studies implicate principal cell axons coupled through gap junctions in their generation. In vivo recordings, however, show a correlation between VFOs and interneuron activity. Roopun et al. studied VFOs in human epileptic tissue to clarify the mechanisms underlying the generation of these waves.

Neocortical tissue slices were taken from areas generating seizure activity in 11 patients undergoing elective neurosurgery for drug-intractable epilepsy. In vitro recordings of spontaneously occurring epileptiform activity in the samples showed that VFOs occurred at the onset of interictal events. According to researchers Mark Cunningham and Miles Worthington, this leads to "a state of pathological hyperexcitability," thereby causally implicating VFOs in seizure generation. VFOs occurred independently of synaptic inhibition mediated by interneurons, and pharmacological manipulation showed that they persisted "after removal of all chemical synaptic transmission ... as long as gap junction conductance [was] high enough.” This finding supports a causal role for gap junction activity rather than interneurons in the generation of VFOs. Current drug treatments do not target this activity.

The identification of this mechanism for VFO generation in human epileptic tissue is promising for treatment. The researchers hope to develop techniques to effectively "target gap junctional intercellular communication ... to prevent the need for radical surgery".

Eleanor Beal

Original article Roopun, A. K. et al. A nonsynaptic mechanism underlying interictal discharges in human epileptic neocortex. Proc. Natl Acad. Sci. USA doi:10.1073/ pnas.0912652107 


\section{CORRECTION}

Nonsynaptic mechanisms for interictal discharges in drug-intractable epilepsy

Eleanor Beal

Beal, E. Nat. Rev. Neurol. 6, 61 (2010); doi:10.1038/nrneurol.2009.223

In the February 2010 issue of Nature Reviews Neurology, the name given for one of the

study authors was incorrect. The author's name should have been Miles Whittington. 